\title{
Rapid and sensitive quantitation of heme in hemoglobinized cells
}

\author{
Jason R. Marcero, Robert B. Piel III, Joseph S. Burch, and Harry A. Dailey \\ Department of Biochemistry and Molecular Biology, Biomedical and Health Sciences Institute, University of \\ Georgia, Athens
}

\begin{abstract}
BioTechniques 61:83-91 (August 2016) doi 10.2144/000114444
Keywords: heme, hemoglobin, in-situ spectroscopy, integrating cavity, pyridine hemochromogen assay, fluorescence heme assay, murine erythroleukemia cells, K562 cells
\end{abstract}

Supplementary material for this article is available at www.BioTechniques.com/article/114444.

Rapid and accurate heme quantitation in the research lab has become more desirable as the crucial role that intracellular hemoproteins play in metabolism continues to emerge. Here, the time-honored approaches of pyridine hemochromogen and fluorescence heme assays are compared with direct absorbance-based technologies using the CLARiTY spectrophotometer. All samples tested with these methods were rich in hemoglobin-associated heme, including buffered hemoglobin standards, whole blood from mice, and murine erythroleukemia (MEL) and K562 cells. While the pyridine hemochromogen assay demonstrated the greatest linear range of heme detection, all 3 methods demonstrated similar analytical sensitivities and normalized limits of quantitation of $\sim 1 \mu \mathrm{M}$. Surprisingly, the fluorescence assay was only shown to be distinct in its ability to quantitate extremely small samples. Using the CLARiTY system in combination with pyridine hemochromogen and cell count data, a common hemoglobin extinction coefficient for blood and differentiating MEL and K562 cells of $0.46 \mu \mathrm{M}^{-1} \mathrm{~cm}^{-1}$ was derived. This value was applied to supplemental experiments designed to measure MEL cell hemoglobinization in response to the addition or removal of factors previously shown to affect heme biosynthesis (e.g., L-glutamine, iron).

Heme is an essential and central component in diverse biological processes ranging from gas metabolism, one-electron chemistry, and bacterial pathogenesis (1-3) to its more recently recognized roles in circadian rhythm and small RNA processing $(4,5)$. From a clinical standpoint, the level of hemoglobin-associated heme in blood and body fluid samples can be diagnostic of many pathological conditions. Here, we have focused on the quantitation of heme in standard hemoglobin solutions, erythroid model cell lines, and blood samples as a benchmark for comparison of currently available technologies.
Traditional strategies for measuring hemoglobin include, but are not limited to, Drabkin's (cyanmethemoglobin) method (6), the pyridine hemochromogen assay (7), the fluorescence heme assay (8), and reverse-phase HPLC (9). With the exception of the cyanmethemoglobin assay, these approaches infer hemoglobin concentrations from measurements of heme. More recently, chemiluminescentbased methods based on horseradish peroxidase biochemistry have also been developed to quantitate total cellular heme (10) and regulatory heme (11). We have previously relied on the pyridine hemochromogen and fluores- cence assays to determine cellular heme and hemoglobin concentrations as described elsewhere $(12,13)$. Fluorescence analysis is a more sensitive technique per se, yet signal emission is complicated by multiple environmental factors and therefore does not permit application of the Beer-Lambert law (14). Like the chemiluminescence method, both pyridine hemochromogen and fluorescence assays involve laborious sample preparations that require the handling and disposal of hazardous chemicals.

As an alternative, the CLARiTY 1000 spectrophotometer by Olis, Inc. (Bogart, GA) has made it possible to

\section{METHOD SUMMARY}

Two traditional methods of heme quantitation, pyridine hemochromogen and fluorescence heme assays, are compared side-by-side to absorbance spectroscopy using the CLARiTY spectrophotometer. The CLARiTY system offers the advantage of direct measurement of heme in intact cells without the need for derivatization and the concomitant handling of hazardous chemicals. Consequently, this approach allows for post-scan processing (e.g., qPCR, immunoblotting) or reculturing of samples. 

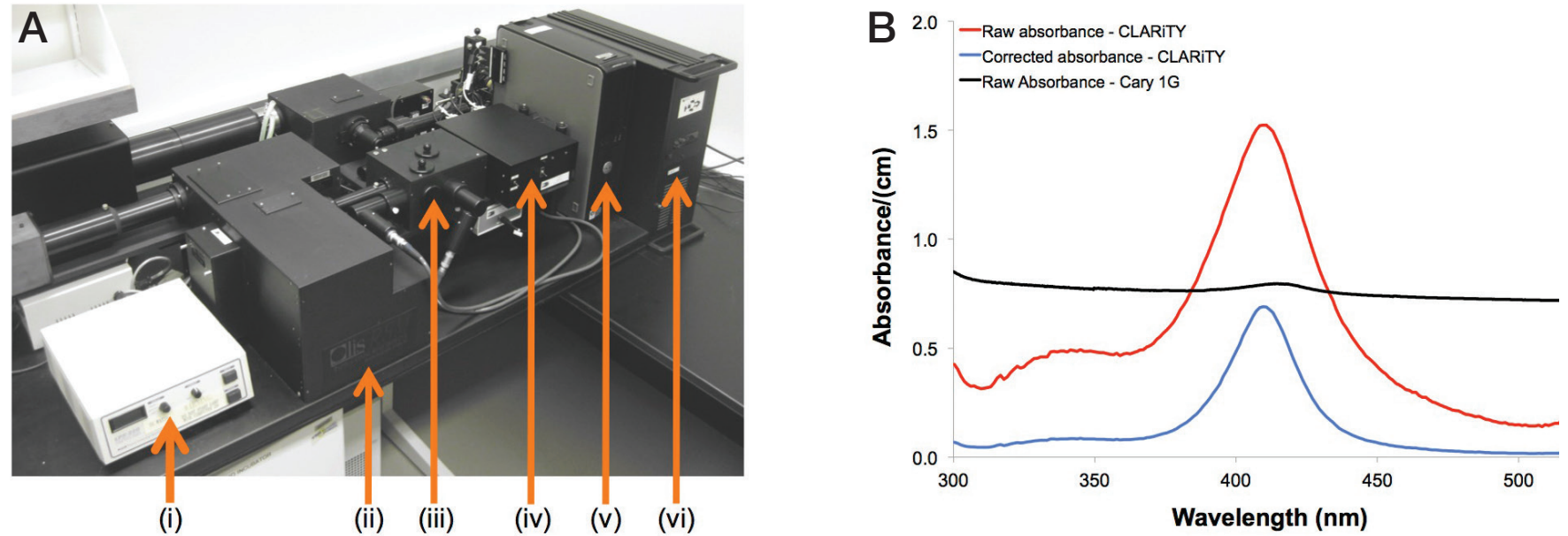

Figure 1. The Olis CLARITY spectrophotometer diffuses light from a rapid scanning monochromator (RSM) to obtain absorbance readings for hemoglobin in turbid whole-cell solutions. (A) Major components of the CLARiTY system include: (i) xenon arc lamp with igniter, (ii) RSM, (iii) CLARiTY module with 1- and 10-mL cuvettes adjoined to separate photomultiplier tubes, (iv) control box to switch scan settings between RSM and CLARiTY, (v) computer hard drive, and (vi) electronic box. (B) Overlay of absorbance spectra for MEL cells washed and resuspended in 1× PBS following a 72-h induction in regular media containing 1.5\% DMSO. The apparent absorbance scan of a 1-mL cell sample on the RSM-CLARiTY is decreased upon Fry correction (19) for the increased pathlength within the cuvette. A traditional transmission spectrophotometer (Cary 1G by Varian) was used to scan a 1:10 dilution of the same cell resuspension analyzed on the CLARiTY.

measure hemoglobinization in turbid whole-cell suspensions quickly and without sample derivatization. This technology has been implemented in situ elsewhere (15-18), and here we demonstrate use of the CLARITY system (Figure 1A) to quantitate heme in buffered blood and cell culture resuspensions. The key component of the CLARITY is a novel integrating cavity absorption meter (ICAM) that contains a quartz cuvette surrounded by a highly reflective coating. UV/visible light bracketing the heme Soret band is generated by a rapid-scanning monochromator (RSM) and directed into the cuvette to produce isotropic, fully diffused light that is subsequently altered only by sample absorption. The light is trapped within the reflective confines of the cuvette until it encounters the output port; thus, its effective pathlength is increased significantly. The enhanced pathlength is inversely related to the detected (apparent) sample absorbance, and this nonlinear effect is corrected by converting the apparent absorbance to absorbance per centimeter using first principles and the Fry Equation (19). The resulting sensitivity of the CLARiTY to light absorption in turbid samples is overwhelmingly superior to that of traditional transmission spectrophotometers (Figure 1B).

Here, we assess and validate our protocol for hemoglobin quantitation using the CLARITY system in a side-by- side comparison to pyridine hemochromogen and fluorescence heme assays of standard hemoglobin solutions and blood samples. Pyridine hemochromogen and CLARITY assays are further employed to analyze hemoglobinization in cultures of differentiating MEL and K562 cells. Specifically, we demonstrate the use of heme concentrations from pyridine hemochromogen spectra to calculate red blood cell indices for mouse blood and cell culture samples, a heme proportionality constant for the fluorescence assay, and a micromolar hemoglobin extinction coefficient for the CLARITY. Additional tests of the CLARITY verify the ability of our system to determine the effects of oxygen, glutamine, iron, aminolevulinic acid (ALA), and succinylacetone (SA) on hemoglobin levels in MEL cells. In general, we expect the protocol described here to be useful for heme quantitation in many non-erythroid cell types as well. One exception is plant cells, in which the absorbance signatures of chlorophylls and carotenoids significantly overlap the heme Soret peak.

\section{Materials and methods}

Hemoglobin standards and mouse blood

Lyophilized hemoglobin from bovine blood (Sigma-Aldrich, St. Louis, MO) was dissolved in phosphate buffered saline (1x PBS) to a concentration of 20 $\mu \mathrm{M}$ and diluted for use as standards. Whole mouse blood samples were generously provided by L. Wang (University of Georgia, Athens, GA). Samples were collected in $\mathrm{K}_{2}$ EDTA tubes from adult wild-type C57BL/6 mice (one female and one male) and subsequently diluted between 1:250 and 1:100,000 in 1x PBS and kept on ice.

\section{Cell culture}

Cells from the MEL strain DS19 (20) and K562 cell line ATCC CCL-243, originally established by Lozzio and Lozzio (21), were maintained at $37^{\circ} \mathrm{C}$ and $5 \% \mathrm{CO}_{2}$ in complete media consisting of DMEM with $25 \mathrm{mM}$ glucose and $1 \mathrm{mM}$ sodium pyruvate (Cellgro, Corning, NY) supplemented with $2 \mathrm{mM}$ L-glutamine, 9\% (v/v) FBS (Atlanta Biologicals, Atlanta, GA), and $1 \times$ Pen/Strep (Cellgro). K562 cells were seeded at $1 \times 10^{5} \mathrm{cells} / \mathrm{mL}$ and induced to differentiate in complete media containing $1 \mathrm{mM}$ sodium butyrate (Sigma-Aldrich). MEL cultures were seeded at $2.5 \times 10^{5} \mathrm{cell} / \mathrm{s} / \mathrm{mL}$ and induced in media with $1.5 \%(\mathrm{v} / \mathrm{v}) \mathrm{DMSO}$ (Sigma-Aldrich). Multi-day induction courses of MEL and K562 cells were carried out in triplicate. In addition, MEL cells were induced for 72 or $96 \mathrm{~h}$ under each of the following sets of conditions: (i) 0-4 mM L-glutamine (Cellgro); (ii) 5\% and $20 \% \mathrm{O}_{2}$; (iii) $100 \mu \mathrm{M}$ iron as iron dextran (Fisons, UK) and/or $100 \mu \mathrm{M}$ 
Table 1. Detection parameters and assay information for hemoglobin standard solutions.

\begin{tabular}{|c|c|c|c|c|c|c|c|}
\hline Method & $\begin{array}{l}\mathbf{L O D}_{\mathrm{R}}, \mathrm{LOD}_{\mathrm{N}} \\
(\mu \mathrm{M})\end{array}$ & $\begin{array}{l}\mathrm{LOQ}_{\mathrm{R}}, \mathrm{LOQ}_{\mathrm{N}} \\
(\mu \mathrm{M})\end{array}$ & $\begin{array}{l}\mathrm{LOL}_{\mathrm{R}}, \mathrm{LOL}_{\mathrm{N}} \\
\quad(\mu \mathrm{M})\end{array}$ & $\begin{array}{c}\text { Analytical sensitivity } \\
\qquad\left(\mu \mathrm{M}^{-1}\right)\end{array}$ & Sample volume (mL) & $\begin{array}{l}\text { Requires sample } \\
\text { derivatization? }\end{array}$ & $\begin{array}{l}\text { Approximate time for } \\
1,96 \text {-cell assay(s) }\end{array}$ \\
\hline CLARITY & $\begin{array}{c}0.0362 \\
0.0362\end{array}$ & $\begin{array}{l}1.07 \\
1.07\end{array}$ & $\begin{array}{l}4.26 \\
4.26\end{array}$ & 30.8 & 1 & No & $1 \mathrm{~min}, 1.5 \mathrm{~h}$ \\
\hline
\end{tabular}

ALA (Sigma-Aldrich); and (iv) $500 \mu \mathrm{M}$ SA (Sigma-Aldrich). Hypoxic cultures were grown in a sealed incubator flushed with $5 \% \mathrm{O}_{2} / 5 \% \mathrm{CO}_{2} /$ balance $\mathrm{N}_{2}$ at $37^{\circ} \mathrm{C}$.

\section{Cell counting}

Mouse red blood cells (1:500 dilution in $1 \times$ PBS) were counted on a hemocytometer. MEL and K562 cells were washed and diluted in $1 \times$ PBS before cell counts were done with a Scepter handheld cell counter (Millipore, Billerica, MA) as previously described (22). MEL and K562 cell proliferation values were obtained by dividing the ratio of live cells at the end of each time course to the number of cells seeded at the beginning by the same ratio for untreated cultures.

\section{Spectrophotometric hemoglobin} quantitation using the CLARiTY

Absorbances for hemoglobin standards and whole-cell suspensions were measured in the Olis CLARiTY 1000A spectrophotometer with an integrated RSM-ICAM containing a 1-mL cuvette. A total of 10 scans from $280-520 \mathrm{~nm}$ were collected per sample. Apparent absorbance values for $1-\mathrm{mL}$ samples were recorded relative to a $1 \times$ PBS baseline at the heme Soret peaks of $400 \mathrm{~nm}$ for the standard solutions (which contained methemoglobin) and $410 \mathrm{~nm}$ for cell samples. Fry correction (19) was carried out to normalize enhanced pathlength values to $1 \mathrm{~cm}$ using SpectralWorks software (Olis, Inc.). The Soret peak baseline was then determined for the corrected spectra by linear interpolation. Heme concentrations were obtained from pyridine hemochromogen assays and combined with Fry-corrected absorbance values from the CLARITY to define a hemoglobin extinction coefficient for mouse blood and MEL and K562 cell cultures. The assumptions that all cellular heme in these samples is bound to hemoglobin and that each hemoglobin tetramer contains four heme molecules were made. The mean corpuscular hemoglobin $(\mathrm{MCH})$ was then calculated in picograms per cell according to the following form of the Beer-Lambert law,

$$
M C H=\frac{M_{H b} A_{F r y}}{\varepsilon_{H b} N}
$$

where $M_{H b}=$ molecular weight of hemoglobin $(64.5 \mathrm{kDa}), A_{\text {Fry }}=$
Fry-corrected absorbance (absorbance $/ \mathrm{cm}$ ) at $410 \mathrm{~nm}, \boldsymbol{\varepsilon}_{\mathrm{Hb}}=$ hemoglobin extinction coefficient, and $N=$ absolute number of cells.

\section{Pyridine hemochromogen assay}

Samples of mouse blood and cell culture resuspensions were kept on ice and disrupted by sonication. An equal volume of $50 \%(\mathrm{v} / \mathrm{v})$ pyridine : $0.2 \mathrm{~N} \mathrm{NaOH}$ solution was added to the hemoglobin standards or homogenized cell samples to prepare the pyridine hemochrome derivative. Heme content was measured from reduced minus oxidized difference spectra as described by Berry and Trumpower (23) and based on the original work of Paul et al. (7). Scans were carried out on a Cary 1G UV-visible spectrophotometer, whose settings were controlled using Cary WinUV software (Agilent, Santa Clara, CA). The absorbance difference between the reduced peak at $556 \mathrm{~nm}$ and oxidized trough at $540 \mathrm{~nm}$ (Supplementary Figure S1A) was converted to heme concentrations using the extinction coefficient $23.98 \mu \mathrm{M}^{-1} \mathrm{~cm}^{-1}$ (23).

\section{Fluorescence heme assay}

Fluorescence assays were carried out as previously published $(8,13)$, with minor modifications. Briefly, aliquots of hemoglobin standards or sonicated cells were diluted 40 -fold in concentrated oxalic acid and then split. One sample was boiled at $99^{\circ} \mathrm{C}$ for $30 \mathrm{~min}$ in a Robocycler PCR machine (Stratagene, La Jolla, CA). The other solution was treated in the same way except that it was maintained at room temperature throughout to control for the presence of endogenous porphyrins. All samples were then analyzed on a CLARIOstar microplate reader (BMG LABTECH, Offenburg, Germany) at excitation and emission wavelengths of $400 \mathrm{~nm}$ and $662 \mathrm{~nm}$, respectively. 
A

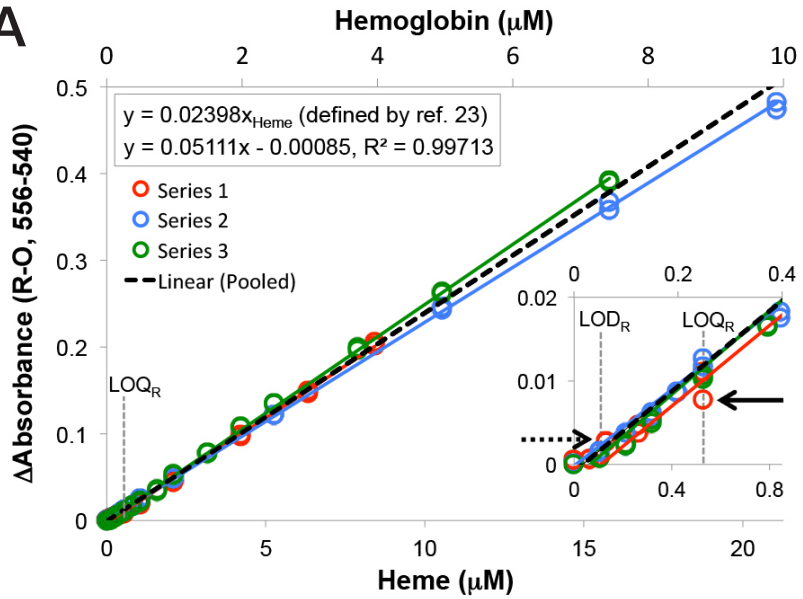

B

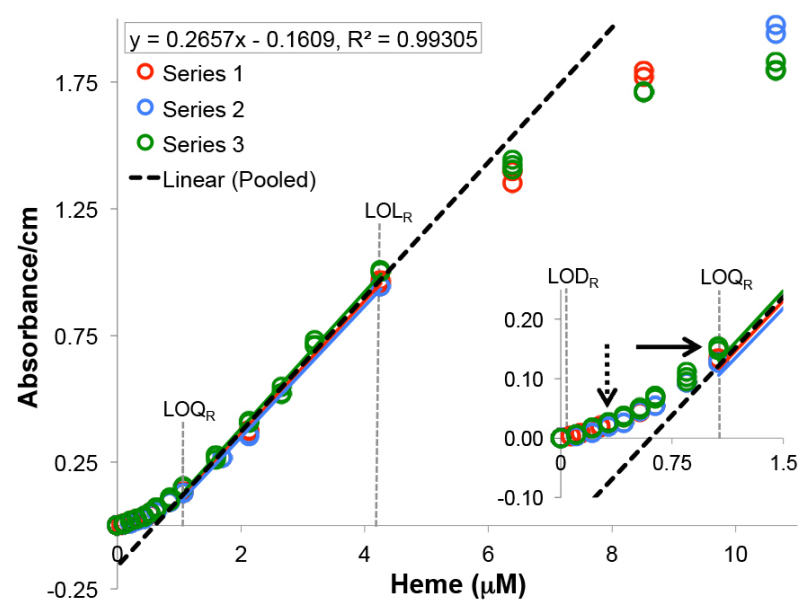

C

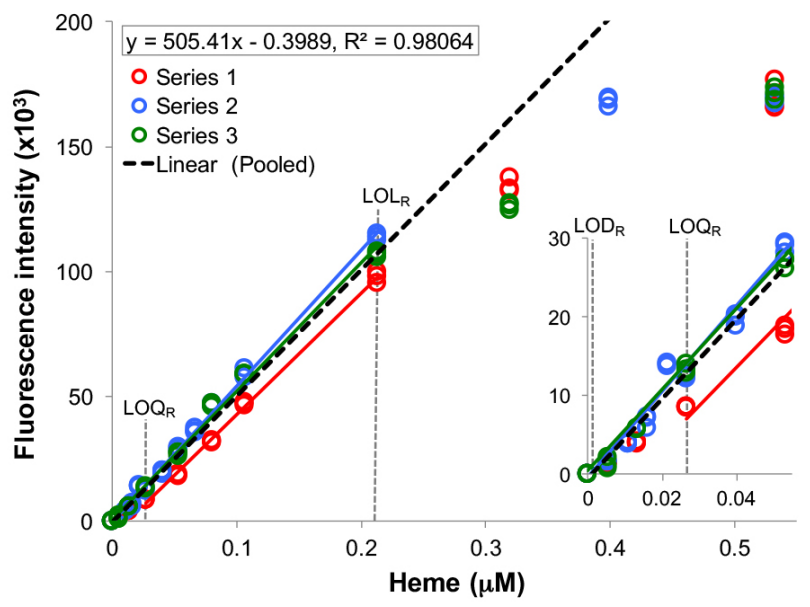

Figure 2. Determination of detection parameters for pyridine hemochromogen, CLARiTY, and fluorescence assays of standard hemoglobin dilutions. (A) Pyridine hemochromogen and (B) CLARiTY calibration curves. Scans for select points (see arrows) in the $L O D_{p}-L O Q_{p}$ range can be found in the Supplementary Material (Supplementary Figure S1, A and B). (C) Fluorescence calibration line. Inset plots magnify the region near the origin. Each method was carried out on three separate days (Series 1, 2, and 3) with two or three repeat measurements per day. Slopes of the linear regions of the pyridine hemochromogen and CLARITY curves represent micromolar extinction coefficients for pooled data in each type of analysis. LOD: limit of detection; LOQ: limit of quantitation; LOL: limit of linearity; subscript $R=$ value in terms of the raw sample concentration.
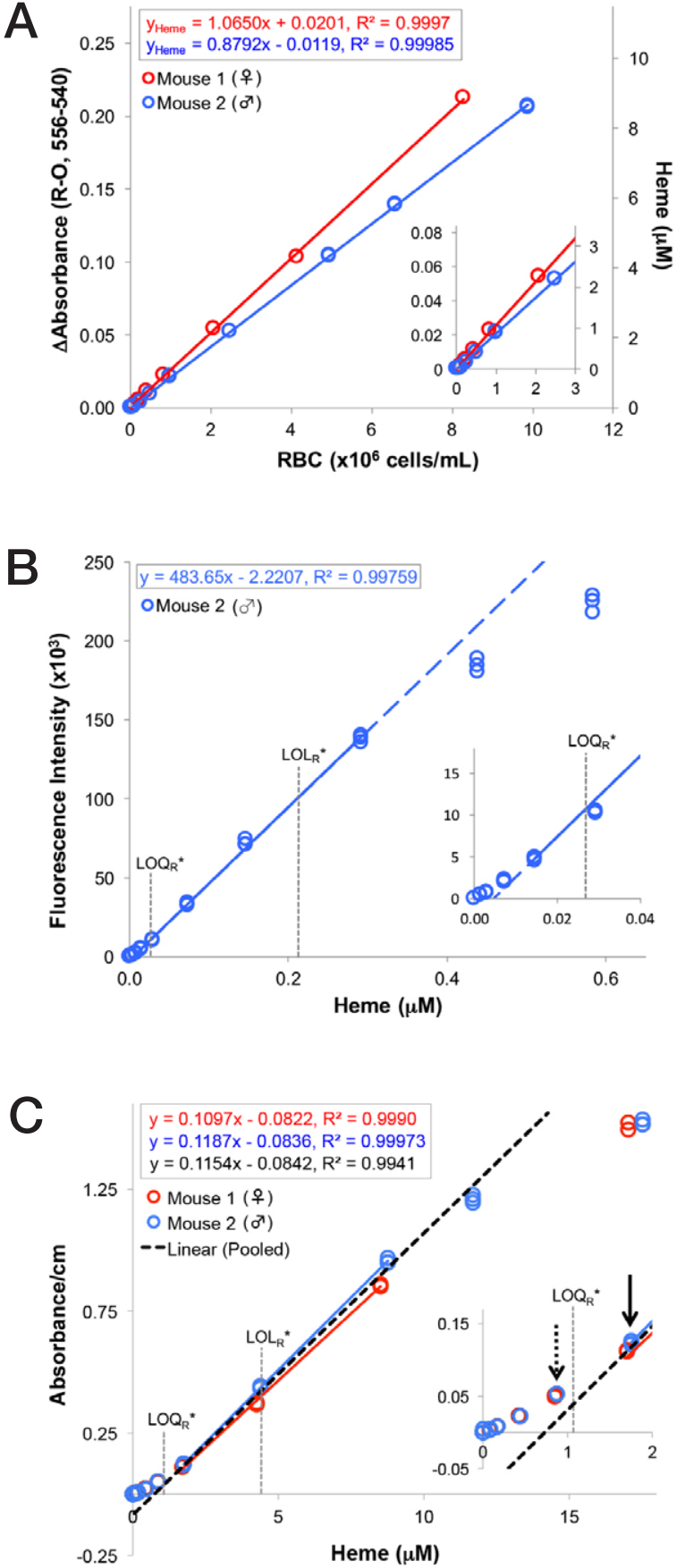

Figure 3. Pyridine hemochromogen, CLARITY, and fluorescence assays of whole mouse blood. Dilutions of clot-free blood (1:250-1:150,000) were prepared in 1× PBS and analyzed for 2 mice (Mouse 1: female, Mouse 2: male). (A) Pyridine hemochromogen absorbance differences were measured twice for each mouse. The corresponding heme concentrations were plotted against the number of red blood cells counted on a hemocytometer. Slope values are equivalent to heme content (fmol/cell). Scans maintained the same profile seen in Supplementary Figure S1A. (B) Fluorescence assays were carried out with three replicates for each dilution of Mouse 2 blood. (C) CLARiTY analysis produced statistically different extinction coefficients for heme in Mouse 1 and Mouse 2. Individual data points bracketing the $L O Q_{R}$ (see arrows) reveal a Soret peak shift from $400 \mathrm{~nm}$ in the hemoglobin standards (Supplementary Figure S1B) to $410 \mathrm{~nm}$ in blood (Supplementary Figure S1C). This $10 \mathrm{~nm}$ difference was not unexpected, as the lyophilized hemoglobin solid used in the standards contained a significant amount of methemoglobin. Inset plots magnify the region near the origin. * indicates a limit parameter established for purified hemoglobin standards (Figure 2), shown for comparison. LOQ: limit of quantitation; subscript $R$ : value in terms of the raw sample concentration. 
Table 2. Mouse whole blood indices and spectroscopic constants.

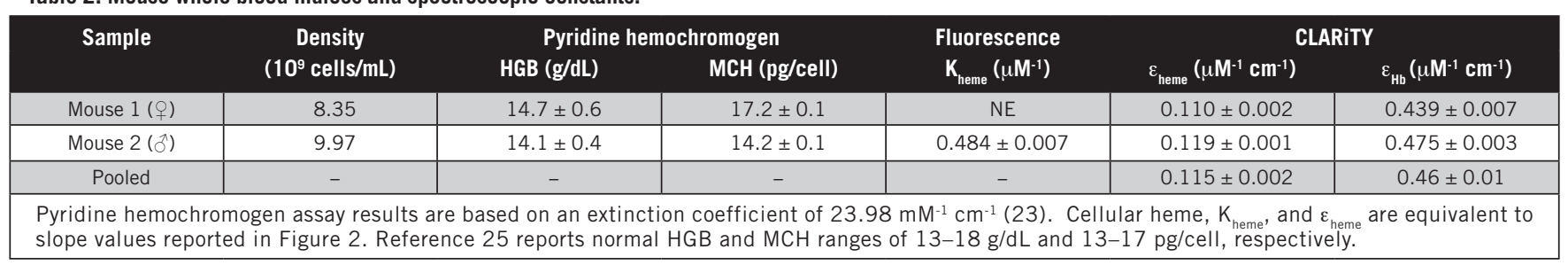

Sensitivity and limits of detection and quantitation

Calibration data for hemoglobin standards were pooled to derive a limit of detection (LOD) for the CLARITY, pyridine hemochromogen assay, and fluorescence heme assay using the limit-of-blanks approach summarized elsewhere (24). Limits of quantitation $(L O Q)$ and upper limits of linearity (LOL) were also determined. The LOQ was defined as the lowest concentration at which the calibration curve is linear and the coefficient of variance (CV) of replicate samples is $<20 \%$. The LOL was identified as the highest concentration of standard that remained in the linear range of the curve. Limits were established for raw sample concentrations (e.g., $L^{\circ O Q_{R}}$ ) and normalized to original, pre-derivatized concentrations (e.g., $L O Q_{N}$ ). Finally, analytical sensitivity was defined as the slope of the linear region of the curve divided by the standard deviation of the response variable.

\section{Statistics}

Extinction coefficients and red blood cell indices for mouse blood and cell culture samples were determined as mean \pm SD. Statistical significance between slope values was evaluated with GraphPad Prism 6 (GraphPad Software, LaJolla, CA). Significance between two sets of cell culture conditions was established with the two-tailed Student's $t$-test. Multiple treatments were analyzed with one-way ANOVA followed by Tukey's post-hoc analyses using Statistica freeware (StatSoft, Tulsa, OK).

\section{Results and discussion}

Detection parameters in pyridine hemochromogen, CLARITY, and fluorescence assays of hemoglobin standards

Hemoglobin standards were used to derive calibration curves and to compare the detection parameters for the pyridine hemochromogen, CLARiTY, and fluorescence assays, including LOD, LOQ, LOL, and analytical sensitivity. The pyridine hemochromogen assay served a dual purpose in that the results were also used to check the heme content of the commercially available hemoglobin, which was not expected to contain four bound heme molecules per hemoglobin tetramer due to impurities, the unknown impact of oxidation (i.e., conversion to methemoglobin), and molecular structure deformities inherent to the lyophilization process. Based on a comparison of slope values from pooled plots of reduced minus oxidized absorbances against both hemoglobin and heme concentrations (Figure 2A), it was found that the lyophilized hemoglobin contained 2.13 heme groups per hemoglobin tetramer. The detection parameters listed in Table 1 were defined in terms of heme concentrations using this value.

Corrected CLARITY absorbances for the hemoglobin standards at 400 nm (Figure 2B, Supplementary Figure
S1B) were pooled and yielded a heme extinction coefficient (slope) of 0.266 $\pm 0.004 \mu \mathrm{M}^{-1} \mathrm{~cm}^{-1}$. The CLARiTY calibration curve was sigmoidal in shape, and the linear range of response was determined by maximizing $R^{2}$ values between visually identified bend points in the curve (Table 1). Although the $L_{O} D_{R}$ for the CLARITY was approximately one-third the $L O D_{R}$ for the pyridine hemochromogen assay, the $L O Q_{R}$ for the CLARITY was more than twice the $L O Q_{R}$ for pyridine hemochromogen. Because the $\mathrm{CV}$ dropped below $20 \%$ at $0.11 \mu \mathrm{M}$ heme, we defined $L O Q_{R}$ for the CLARITY system by the lowest heme concentration in the linear region of the curve. This 2-fold difference in $L O Q_{R}$ was mirrored closely by the analytical sensitivities of the two methods.

Fluorescence heme assays also generated nonlinear calibration data across the range of standard concentrations (Figure 2C). However, unlike the CLARITY, two of the three series maintained linearity through the origin. The slope of the line $\left(505 \pm 8 \mu \mathrm{M}^{-1}\right)$ is referred to as $\mathrm{K}_{\text {heme, }}$, a proportionality constant in the expression,

$$
I_{F}=K_{\text {heme }} C_{\text {heme }}
$$

[Eq. 2],

where $I_{F}=$ fluorescence intensity and $\mathrm{C}_{\text {heme }}=$ heme concentration. The $\mathrm{K}_{\text {heme }}$ constant is a complex term that is directly proportional to the excitation beam intensity, quantum yield, heme extinction coefficient $\left(\varepsilon_{\text {heme }}\right)$, and path

Table 3. Cell culture heme and hemoglobin properties.

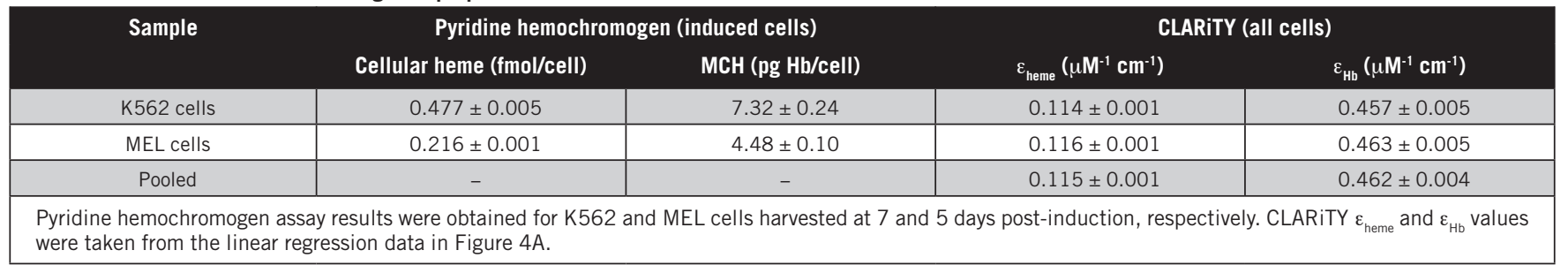


A

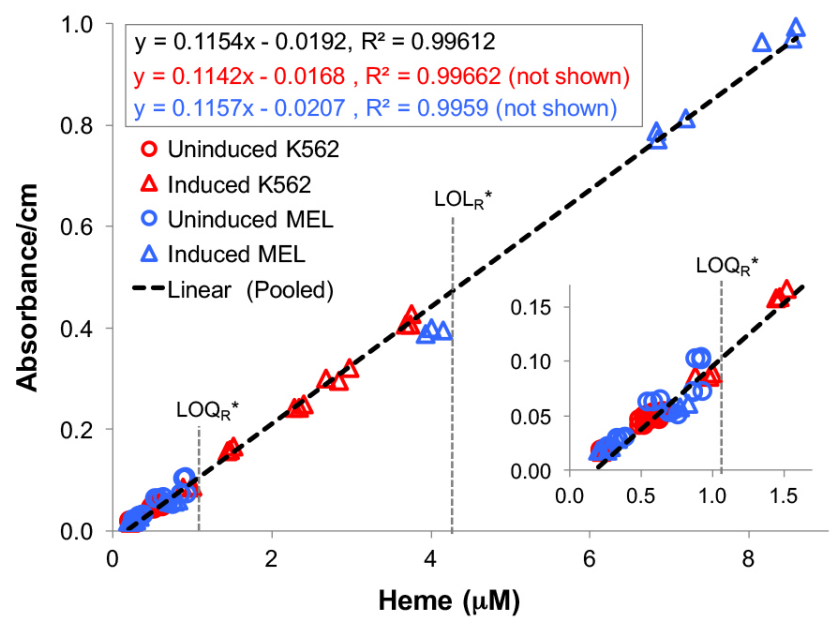

$\mathrm{B}$

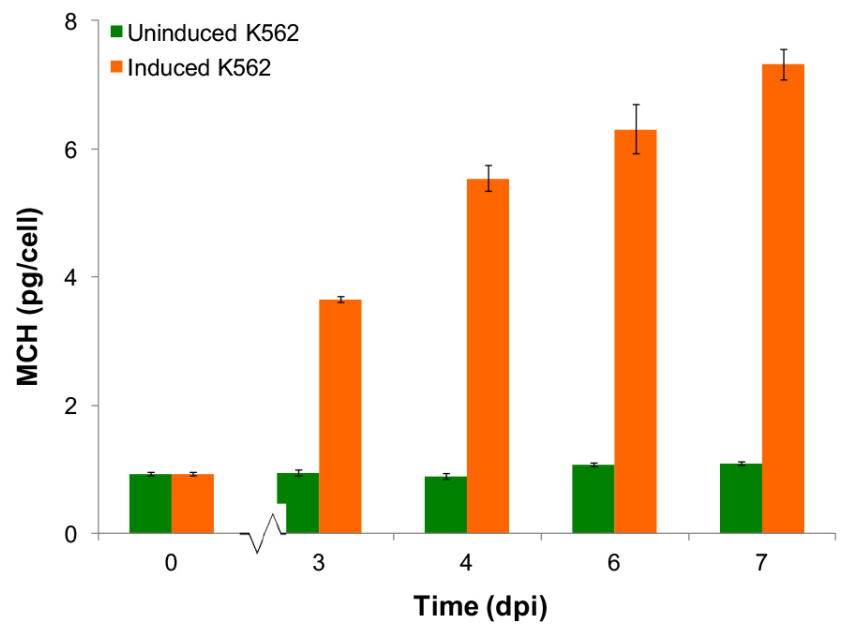

C

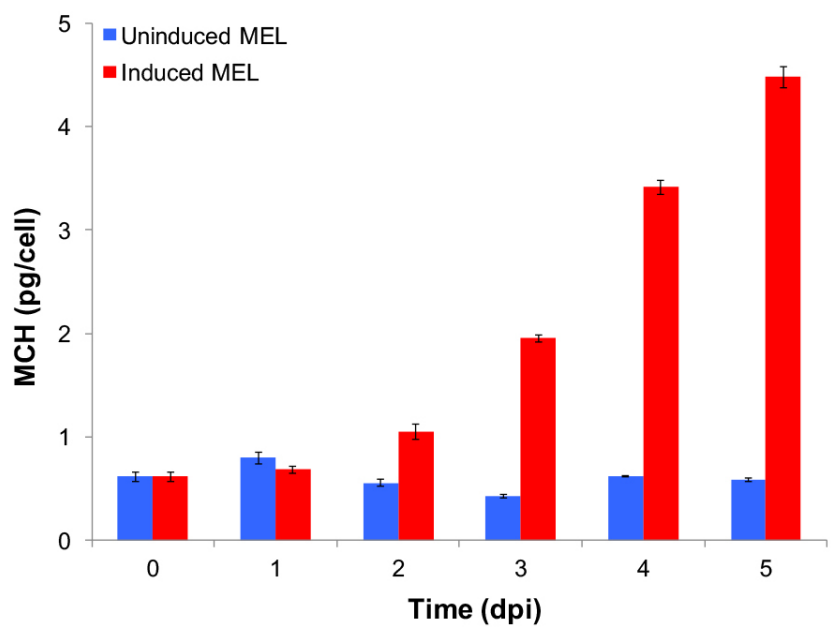

Figure 4. Determination and application of a micromolar extinction coefficient for whole-cell K562 and MEL suspensions. Data from CLARiTY and pyridine hemochromogen assays were used in combination (A) to derive $\mathrm{MCH}$ values for induced and uninduced K562 (B) and MEL (C) cell cultures over 7- and 5-day time courses, respectively (dpi $=$ days post induction). The regression line for pooled absorbance/cm values (CLARITY) and heme concentrations (pyridine hemochromogen assay) (A) was used to a derive a single heme extinction coefficient because individual K562 and MEL cell slopes (lines not shown for clarity) were not significantly different $(P=0.115)$. Note that points that fell outside the linear range determined for hemoglobin standards $\left(\mathrm{LOQ}_{\mathrm{R}}{ }^{*}-\mathrm{LOL}_{\mathrm{R}}{ }^{*}\right)$ were included in the regression analysis. Error bars represent $\pm \mathrm{SD}$. length (14). This relationship holds at absorbances below 0.05, which can be assumed with the 40 -fold dilution of the standard solutions prior to analysis. The analytical sensitivity of the fluorescence assay was unexpectedly close to the sensitivity of the CLARiTY and less than half the sensitivity of the pyridine hemochromogen assay (Table 1). Analytical sensitivity is dependent on statistical variance and is thus indicative of changes in fluorescence signal with environmental conditions. Surprisingly, the CLARITY demonstrated a lower normalized LOD $\left(\operatorname{LOD}_{N}\right)$ than the fluorescence assay. That is, given at least $1 \mathrm{~mL}$ of sample, the CLARITY and pyridine hemochromogen assays are superior to the fluorescence assay at low concentrations. At higher concentrations, the greater linear range and analytical sensitivity of the pyridine hemochromogen assay make it the preferred approach. Indeed, the fluorescence assay protocol employed here is only unmatched in its ability to quantitate extremely small samples.

Heme and hemoglobinization in mouse blood

As with the hemoglobin standards, the pyridine hemochromogen assay was used to determine heme levels in mouse blood dilutions (Figure 3A). Blood was collected from one male and one female C57BL/6 mouse on separate days and then analyzed (Table 2 ). With the exception of the female $\mathrm{MCH}(17.2$ $\mathrm{pg} / \mathrm{cell}), \mathrm{HGB}$ and $\mathrm{MCH}$ values for both mice fell well within the normal ranges for these indices (25). Considering established inaccuracies in the hemocytometer relative to automated means (26), the elevated female $\mathrm{MCH}$ was likely due to an erroneously low cell count.

Plots of fluorescence (Figure 3B) and corrected CLARITY absorbance at $410 \mathrm{~nm}$ (Figure 3C, Supplementary Figure S1C) against heme concentration generated slope values equivalent to $\mathrm{K}_{\text {heme }}$ and $\varepsilon_{\text {heme }}$, respectively (Table 2). While we expected $\varepsilon_{\text {heme }}$ values for the CLARITY to be the same for both mice, the lower value for the female mouse could also be explained by a lower than actual cell count. Regardless of the deviation, assuming that hemoglobin is saturated with heme and that it is 
the only hemoprotein present in the cells allows us to calculate $\varepsilon_{\mathrm{Hb}}$ as $4 \times$ $\varepsilon_{\text {heme }}$ and directly convert absorbance/ $\mathrm{cm}$ (Beer-Lambert law) or fluorescence intensity (Equation 2) to hemoglobin concentration. In this way, the $\varepsilon_{\text { }}$ for the CLARITY was found to be 0.46 $\pm 0.01 \mu \mathrm{M}^{-1} \mathrm{~cm}^{-1}$. Of further note, visually identified $L O Q_{R}$ values for the CLARITY and fluorescence calibration curves were similar to the values for hemoglobin standards. $\mathrm{LOL}_{\mathrm{R}}$ values, however, were significantly higher than the hemoglobin standards for both methods, suggesting that detection parameters are analyte-specific.

Hemoglobin quantitation in differentiating K562 and MEL cells K562 cells are known to differentiate irreversibly along the erythroid lineage, expressing markers such as glycophorin $A$ and synthesizing unusually large quantities of embryonic and fetal globins when treated with inducing agents such as sodium butyrate (27). MEL cells induced to differentiate by DMSO also exhibit erythroid-specific membrane antigens (28), an increase in globin mRNA (29), and a decrease in cell size (30) not observed in K562 cells.

Applying the same approach used to analyze mouse blood, pyridine hemochromogen assays of MEL and K562 cells that were induced to differentiate with DMSO (5 days) and sodium butyrate (7 days), respectively, or left untreated (uninduced) were used to determine heme concentrations for each culture. Corrected absorbances from the CLARITY plotted against the heme concentrations for MEL and K562 cells generated slope values that were not significantly different between the two cell types (Figure 4A). All samples were thus pooled to derive a single value for $\varepsilon_{\mathrm{Hb}}$ of $0.462 \pm 0.004 \mu \mathrm{M}^{-1} \mathrm{~cm}^{-1}$ (Table $3)$, which is equivalent to $\varepsilon_{\mathrm{Hb}}$ for pooled mouse blood (Table 2) to 2 decimal places. Incidentally, we decided to include cell sample data that fell outside of the defined linear range $\left(L O Q_{R}-L O L_{R}\right)$ for the hemoglobin standards. We felt this was justified for two reasons: (i) no sigmoidal trend was observed in the cell culture analysis, and (ii) the CV for the standards was less than $20 \%$ at 0.11 $\mu \mathrm{M}$ heme, well below the minimum observed sample concentration.
Substitution of $0.462 \mu \mathrm{M}^{-1} \mathrm{~cm}^{-1}$ and cell count numbers into Equation 1 yielded $\mathrm{MCH}$ values. The 7 -day K562 induction time course (Figure 4B) produced a mean maximum $\mathrm{MCH}$ value of $7.31 \mathrm{pg} / \mathrm{cell}$ in induced samples compared to only $0.983 \mathrm{pg} /$ cell in the uninduced controls (Day 7). The induced value is significantly lower than the normal red blood cell level of 27-33 pg/cell (31), which has only been approached previously in K562 cells with glutamine starvation combined with hemin treatment (32). The 5-day MEL induction time course (Figure 4C) resulted in a mean maximum $\mathrm{MCH}$ of $4.47 \mathrm{pg} / \mathrm{cell}$ in induced cultures and $0.583 \mathrm{pg} / \mathrm{cell}$ in uninduced cultures (Day 5). These values indicate that cellular hemoglobin levels are much lower in MEL cells than K562 cells; however, this is not unexpected as the average volume of a $\mathrm{K} 562$ cell is more than three times that of an MEL cell.

The results of additional studies that validate the ability of the CLARITY system to quantitate hemoglobin in MEL cells under various conditions can be found in the Supplementary Material. Specifically, the effects of hypoxia (Supplementary Figure S2), glutamine starvation (Supplementary Figure S3), iron and ALA supplementation (Supplementary Figure S4A), and SA treatment (Supplementary Figure S4B) on heme production and cell proliferation are described.

The CLARITY, pyridine hemochromogen, and fluorescence assays each have unique strengths and weaknesses for the measurement of hemoproteins. The pyridine hemochromogen assay has the largest linear range of operation and the highest analytical sensitivity, but it is also the most labor-intensive of the three approaches. The ability of the fluorescence assay to detect extremely low concentrations of heme is well-known, yet the processes of derivatization and associated dilution considerably offset this attribute. Additionally, fluorescence requires calibration on a day-to-day basis due to variables such as temperature and lamp intensity. In contrast, the consistency of the CLARITY between sample types was clearly established here in defining equivalent $\varepsilon_{\mathrm{Hb}}$ values for wholecell resuspensions of mouse blood and differentiating MEL and K562 cells. The CLARiTY also maintained normalized limits of detection and quantitation that were comparable, if not superior, to the other assays. Although the linear range of the CLARITY is smaller than that of the other methods, this parameter appears to be sample-dependent and is significantly larger for whole-cell samples than for standard solutions of hemoglobin. Further, preliminary data collected on the CLARITY shortly before publication of this work indicate that an empirically based absorbance correction method from Javorfi et al. (33) can produce a greater LOL and linear dynamic range than the Fry Equation. Perhaps most importantly, the CLARITY system is able to quantitate protein-associated heme in situ quickly and without sample derivatization or destruction, permitting downstream applications and/or analyses.

\section{Author contributions}

J.R.M., H.A.D., and R.B.P. designed the study. J.R.M. performed the experiments, processed the data, conducted the statistical analysis, and wrote the manuscript. H.A.D. edited the manuscript, provided laboratory resources, and obtained funding. J.S.B. provided technical support and assisted with data processing.

\section{Acknowledgments}

Funding for this project was provided by NIH grant R01-DK096051. We would like to thank A. Medlock, K. Mohler, and T. Dailey for their assistance with the CLARITY system and cell culture studies. We acknowledge the comments and suggestions provided by J. DeSa Lorenz, R. Desa, P. Boxrud, and K. Solntsev from Olis, Inc. during the presubmission process. This paper is subject to the NIH Public Access Policy.

\section{Competing interests}

The authors declare no competing interests.

\section{References}

1. Ponka, P. 1999. Cell biology of heme. Am. J. Med. Sci. 318:241-256.

2. Tsiftsoglou, A.S., A.I. Tsamadou, and L.C. Papadopoulou. 2006. Heme as key regulator of major mammalian cellular functions: molecular, 
cellular, and pharmacological aspects. Pharmacol. Ther. 111:327-345

3. Weinberg, E.D. 1978. Iron and infection. Microbiol. Rev. 42:45-66.

4. Dioum, E.M., J. Rutter, J.R. Tuckerman, G. Gonzalez, M.A. Gilles-Gonzalez, and S.L. McKnight. 2002. NPAS2: a gas-responsive transcription factor. Science 298:2385-2387.

5. Faller, M., M. Matsunaga, S. Yin, J.A. Loo, and F. Guo. 2007. Heme is involved in microRNA processing. Nat. Struct. Mol. Biol. 14:23-29.

6. Drabkin, D.L. and J.H. Austin. 1935. Spectrophotometric Studies II. Preparations from washed blood cells; nitric oxide hemoglobin and sulfhemoglobin. J. Biol. Chem. 112:51-65.

7. Paul, K.G., H. Theorell, and A. Akeson. 1953. The molar light absorption of pyridine ferroprotoporphyrin (pyridine hemochromogen). Acta Chem. Scand. 7:1284-1287.

8. Morrison, G.R. 1965. Fluorometric Microdetermination of Heme Protein. Anal. Chem. 37:11241126.

9. Bonkovsky, H.L., S.G. Wood, S.K. Howell, P.R. Sinclair, B. Lincoln, J.F. Healey, and J.F. Sinclair. 1986. High-performance liquid chromatographic separation and quantitation of tetrapyrroles from biological materials. Anal. Biochem. 155:56-64.

10. Masuda, T. and S. Takahashi. 2006. Chemiluminescent-based method for heme determination by reconstitution with horseradish peroxidase apo-enzyme. Anal. Biochem. 355:307-309.

11. Atamna, H., M. Brahmbhatt, W. Atamna, G.A. Shanower, and J.M. Dhahbi. 2015. ApoHRPbased assay to measure intracellular regulatory heme. Metallomics 7:309-321.

12. Furhop, J.H.S. K.M. 1975. Laboratory methods in porphyrin and metalloporphyrin research. Elsevier Science LTD, Amsterdam, The Netherlands.

13. Sassa, S. 1976. Sequential induction of heme pathway enzymes during erythroid differentiation of mouse Friend leukemia virus-infected cells. J. Exp. Med. 143:305-315.

14. Harris, D.A. and C.L. Bashford. 1987. Spectrophotometry and Spectrofluorimetry - A Practical approach. IRL press, Oxford, United Kingdom.

15. Blake, R.C., 2nd and M.N. Griff. 2012. In situ Spectroscopy on Intact Leptospirillum ferrooxidans Reveals that Reduced Cytochrome 579 is an Obligatory Intermediate in the Aerobic Iron Respiratory Chain. Front. Microbiol. 3:136.

16. Zorz, J.K., J.R. Allanach, C.D. Murphy, M.S. Roodvoets, D.A. Campbell and A.M. Cockshutt. 2015. The RUBISCO to Photosystem II Ratio Limits the Maximum Photosynthetic Rate in Picocyanobacteria. Life (Basel) 5:403-417.

17. Li, T.F., R.G. Painter, B. Ban, and R.C. Blake 2nd. 2015. The Multicenter Aerobic Iron Respiratory Chain of Acidithiobacillus ferrooxidans Functions as an Ensemble with a Single Macroscopic Rate Constant. J. Biol. Chem. 290:1829318303

18. Rana, N., S. McLean, B.E. Mann, and R.K. Poole. 2014. Interaction of the carbon monoxidereleasing molecule $\mathrm{Ru}(\mathrm{CO}) 3 \mathrm{Cl}$ (glycinate) (CORM-3) with Salmonella enterica serovar Typhimurium: in situ measurements of carbon monoxide binding by integrating cavity dual-beam spectrophotometry. Microbiology 160:2771-2779.

19. Fry, E.S., G.W. Kattawar, B.D. Strycker, and P.W. Zhai. 2010. Equivalent path lengths in an integrating cavity: comment. Appl. Opt. 49:575577.

20. Ohta, Y., M. Tanaka, M. Terada, O.J. Miller, A. Bank, P. Marks, and R.A. Rifkind. 1976. Erythroid cell differentiation: murine erythroleukemia cell variant with unique pattern of induction by polar compounds. Proc. Natl. Acad. Sci. USA 73:1232-1236

21. Lozzio, C.B. and B.B. Lozzio. 1975. Human chronic myelogenous leukemia cell-line with positive Philadelphia chromosome. Blood 45:321-334

22. Ongena, K., C. Das, J.L. Smith, S. Gil, and G. Johnston. 2010. Determining cell number during cell culture using the Scepter cell counter. J. Vis. Exp. pii:220.

23. Berry, E.A. and B.L. Trumpower. 1987. Simultaneous determination of hemes $a, b$, and c from pyridine hemochrome spectra. Anal. Biochem. 161:1-15.

24. Shrivastava, A. and V.B. Gupta. 2011. Methods for the determination of limit detection and limit of quantitation of the analytical methods. Chronicles of Young Scientists 2:21-25.

25. Hedrich, H.J. 2012. The Laboratory Mouse. Elsevier, Amsterdam, The Netherlands.

26. Stone, L.R., Gray, D.R., Remple, K., and Beaudet, M.P. 2009. Accuracy and precision comparison of the hemocytometer and automated cell counting methods. FASEB J. 3:827.2.

27. Andersson, L.C., M. Jokinen, and C.G. Gahmberg. 1979. Induction of erythroid differentiation in the human leukaemia cell line K562. Nature 278:364-365.

28. Furusawa, M. and K.P. Takahashi. 1972. Erythrocyte membrane-specific antigens common to several species of rodentia. Nat. New Biol. 235:242

29. Ross, J., Y. Ikawa, and P. Leder. 1972. Globin messenger-RNA induction during erythroid differentiation of cultured leukemia cells. Proc. Natl. Acad. Sci. USA 69:3620-3623.

30. Friend, C., W. Scher, J.G. Holland, and T. Sato. 1971. Hemoglobin synthesis in murine virus-induced leukemic cells in vitro: stimulation of erythroid differentiation by dimethyl sulfoxide. Proc. Natl. Acad. Sci. USA 68:378-382.

31. Vajpayee, N., S.S. Graham, and S. Bem. 2011. Basic examination of blood and bone marrow, p. 509-535. In R.A.a.P. McPherson, M.R. (Ed.), Henry's Clinical Diagnosis and Management by Laboratory Methods. Saunders Elsevier, Philadelphia, PA.

32. Erard, F., A. Dean, and A.N. Schechter. 1981. Inhibitors of cell division reversibly modify hemoglobin concentration in human erythroleukemia K562 cells. Blood 58:1236-1239.

33. Javorfi, T., J. Erostyak, J. Gal, A. Buzady, L. Menczel, G. Garab, and K. Razi Naqvi. 2006. Quantitative spectrophotometry using integrating cavities. J Photochem Photobiol B 82:127-131.

Received 01 March 2016; accepted 20 April 2016.

Address correspondence to Harry A. Dailey, Biomedical and Health Sciences Institute, Paul D. Coverdell Center, 500 DW Brooks Drive, Athens, GA 30602-7394. E-mail: hdailey@uga.edu

To purchase reprints of this article, contact: biotechniques@fosterprinting.com

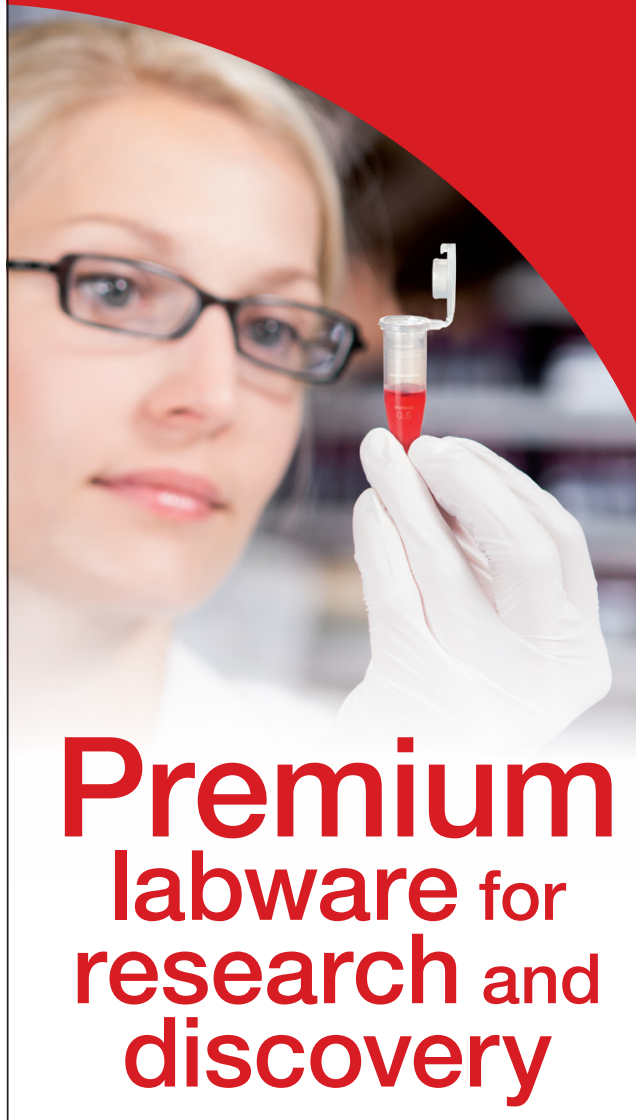

Low-Binding Micro Tubes

- Minimal DNA / protein loss

- SafeSeal locking cap

- Certified PCR-Performance Tested

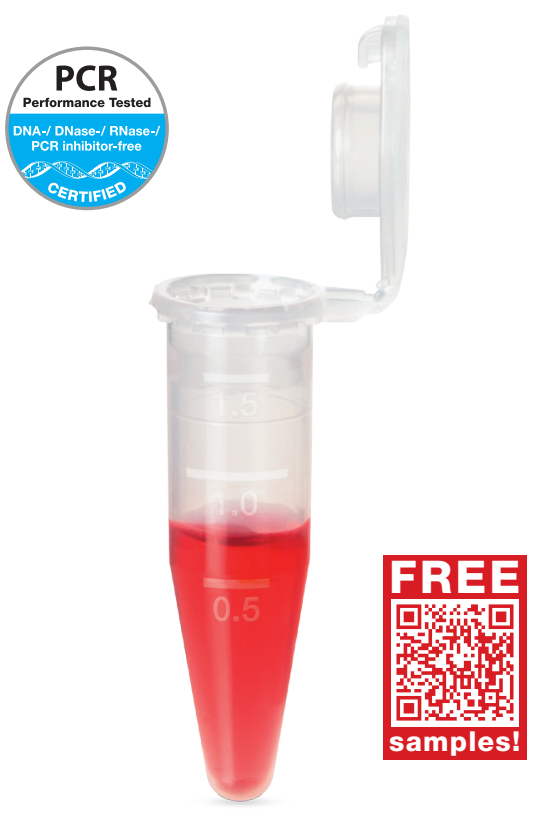

www.sarstedt.com 